\title{
Image Segmentation using Two-Layer Pulse Coupled Neural Network with Inhibitory Linking Field
}

\author{
Heggere S. Ranganath ${ }^{1}$ and Ayesha Bhatnagar ${ }^{2}$ \\ ${ }^{1}$ The University of Alabama in Huntsville \\ Huntsville, Alabama, 35899, USA \\ ranganat@cs.uah.edu \\ ${ }^{2}$ The University of Alabama in Huntsville \\ Huntsville, AL 35899, USA \\ abhatnag@cs.uah.edu
}

\begin{abstract}
For over a decade, the Pulse Coupled Neural Network (PCNN) based algorithms have been used for image segmentation. Though there are several versions of the PCNN based image segmentation methods, almost all of them use singlelayer PCNN with excitatory linking inputs. There are four major issues associated with the single-burst PCNN which need attention. Often, the PCNN parameters including the linking coefficient are determined by trial and error. The segmentation accuracy of the single-layer PCNN is highly sensitive to the value of the linking coefficient. Finally, in the single-burst mode, neurons corresponding to background pixels do not participate in the segmentation process. This paper presents a new 2-layer network organization of PCNN in which excitatory and inhibitory linking inputs exist. The value of the linking coefficient and the threshold signal at which primary firing of neurons start are determined directly from the image statistics. Simulation results show that the new PCNN achieves significant improvement in the segmentation accuracy over the widely known Kuntimad's single burst image segmentation approach. The two-layer PCNN based image segmentation method overcomes all three drawbacks of the single-layer PCNN.
\end{abstract}

Keywords - Image Segmentation, Neural Networks, Pulse Coupled Neural Network, Two-layer PCNN, Image Processing

\section{INTRODUCTION}

The single layered Pulse Coupled Neural Network (PCNN) is a laterally connected two-dimensional array of artificial neurons known as Pulse Coupled Neurons (PCN). Though Eckhorn did not refer to his neuron model as PCN, the artificial neuron model (Eckhorn's neuron) developed by him based on the study of the visual cortex of cats is the first PCN model [1]. By using a laterally connected recurrent network of Eckhorn's neurons, he was successful in emulating some of the neuro-physiological phenomena observed in cat's visual cortex. Ranganath, Kuntimad and Johnson modified Eckhorn's model for image processing applications including image segmentation, smoothing and object detection. They called the simplified model the pulse coupled neuron [2]. In 1999, Kuntimad compared the image segmentation result of the singleburst PCNN algorithm with those of other methods. This was accomplished by segmenting several images consisting of two regions, object and background, in which intensity ranges of object and background regions overlapped significantly. The segmentation result of the PCNN based algorithm was found to be consistently better than the segmentation results obtained by using optimal thresholding, region growing, split-and merge, and probabilistic relaxation algorithms [3]. It has been proved that the single-burst PCNN can segment a two-region image perfectly even if the two intensity ranges overlap significantly when there exist linking coefficient and linking radius values (PCNN parameters) for which two inequalities involving linking coefficient, linking radius, object pixel intensity range, and background pixel intensity range are consistent [4]. However, no method has been suggested for the automatic determination of the two parameters from image statistics. Karvonen used the PCNN to segment Baltic sea ice Synthetic Aperture Radar (SAR) images [5]. He estimated the Gaussian probability density function that best represented the intensity distribution of the pixels of each region, and calculated the primary firing threshold and the linking coefficient for each region. Though his approach may have suited to segment Baltic ice SAR images it is not expected to give satisfactory result when region pixel intensity distribution is not Gaussian. In many cases, the image histogram may not even be multi-modal. Therefore, automatic determination of primary firing thresholds and the corresponding linking coefficient values is still an open problem. The PCNN based image segmentation process can be viewed as a region growing method where seed pixels are identified by the primary firing neurons and the region growing is accomplished by capturing spatially connected neighbouring neurons through secondary firing. Stewart et. al. have used PCNN to develop a seeded region growing method in which seed locations are internally generated [6]. They have avoided the difficulty of choosing optimal value for the linking coefficient for each region by gradually incrementing the value of the linking coefficient to grow the region in multiple steps. The process terminates when at least one of the three termination conditions is satisfied.

A few researchers have used the PCNN with the original Eckhorn's neuron model to segment images [7-9]. Note that Eckhorn's neuron consists of a feeding receptive field, a linking receptive field, and a spike or pulse generator. The spike generator has one leaky integrator, the output of which is the threshold signal. The feeding and linking receptive fields have several leaky integrators. Each leaky integrator has two parameters, amplitude and decay time constant. Even if we assume that all leaky integrators in each receptive field are identical, there are six parameters. The linking coefficient is the seventh parameter. It is obvious that selection of appropriate values for these parameters is a challenging 
problem. Usually these parameter values are determined by trial and error. Also, when Eckhorn's model is used a neuron is allowed to pulse more than once during the segmentation process. Ma et. al. have allowed the pulsing activity to continue for a large number of iterations (a few thousand). At the end of each iteration the entropy of the segmented image is computed. The segmented image of the iteration at which the entropy attains its maximum value is taken as the final result. Based on the experiments conducted, they claim that as entropy increases the details in the segmented image increase [7]. Ma, Liu, and Qian have also suggested that a possible way for automating the selection of the segmentation result is by selecting the result of the iteration for which the discrepancy between the input image and the segmented image as measured by cross-entropy is minimum [8].

\section{IMAGE SEGMENTATION USING SINGLE_LAYER PCNN}

An N X N laterally connected single-layer PCNN was used by Kuntimad and Ranganath to segment an N X N image [3-4]. The neuron $N_{i, j}$ consists of a feeding input $X_{i, j}$ (intensity of its corresponding image pixel), a linking receptive field which gathers linking input $L_{i, j}(t)$ from its 8-neighbors, and a pulse generator as shown in Fig. 1. The internal activity of $N_{i, j}$ is computed by combining the feeding and linking inputs as

$$
U_{i, j}(t)=X_{i, j}\left(1+\beta L_{i, j}(t)\right) \text {. }
$$

where, $\beta>0$ is a parameter known as the linking coefficient. When $U_{i, j}(t)>\theta_{i, j}(t)$, the neuron $N_{i, j}$ fires $\left(Y_{i, j}(t)=1\right)$ and sends linking input to each of its 8-neighbors through a linking leaky integrator $(L L I)$, and also charges the threshold signal generator to a very high value $\theta_{\max }$ to ensure that $N_{i, j}$ will not fire again in the current pulsing cycle [3]. One may prefer to use unit linking and avoid the use of leaky integrators in the linking receptive field.

Consider an image of two regions, object and background. Assume that the object is brighter than the background. Let (Bmin, $B \max$ ) and (Omin, Omax) be intensity ranges of the background and object pixels such that the object is brighter. If Bmax > Omin then the two intensity ranges overlap, and perfect segmentation becomes difficult to achieve. In fact, the

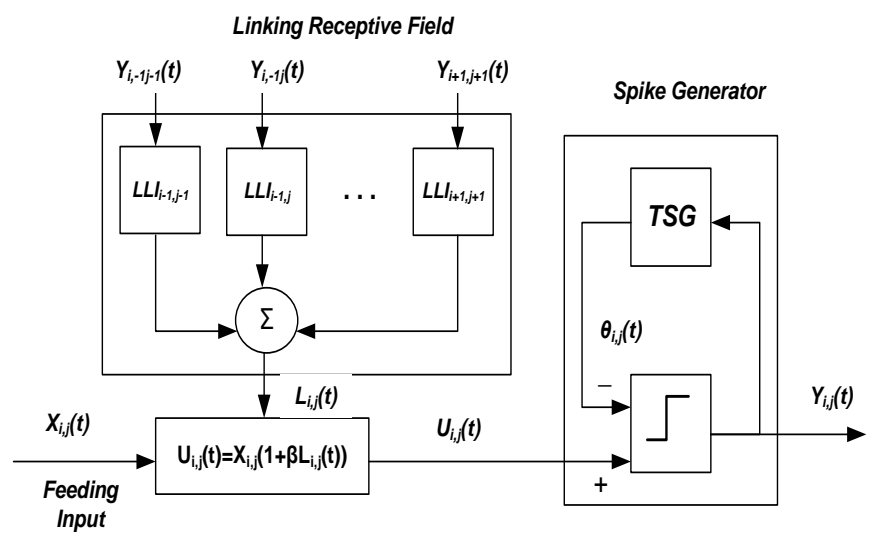

Fig. 1 The Pulse Coupled Neuron Model

number of pixels incorrectly assigned increases as the extent of overlap increases. But, PCNN segments such images perfectly if the following two inequalities are satisfied.

1) The neurons corresponding to the object pixels with intensity Omax pulse naturally (primary pulsing without the help of linking input) at time $t=T($ Omax $)$ where $T(O \max )$ is the time required for the threshold signals to decay from their maximum value of $\theta_{\max }$ to Omax.
2) During secondary firing due to fast linking, all object neurons for which the following inequality is true are captured.

$$
X_{i, j}\left(1+\beta L_{i, j}(T(\text { Omax })) \geq \operatorname{Omax}\right.
$$

In the above inequality, $\beta$ is the linking coefficient, $L_{i, j}(T($ Omax $))$ is the total linking input received by $N_{i, j}$ from its 8-neighbors, and $X_{i, j}$ is the intensity of pixel $(i, j)$ which is the feeding input to $N_{i, j}$.

3) Similarly, during secondary firing, all background neurons for which the following inequality is not true are also captured.

$$
X_{p, q}\left(1+\beta L_{p . q}(T(\text { Omax }))<\right.\text { Omax }
$$

If it is possible to find a value of $\beta$ for which the inequality (2) is true for all object neurons and the inequality (3) is true for all background neurons, then only the object neurons can be made to pulse together at $T(\mathrm{Omax})$ and thus leading to perfect segmentation. When perfect segmentation is not possible the goal is to capture maximum number of object neurons and minimum number of background neurons as possible.

It is obvious that inequalities (2) and (3) impose opposing conditions on $\beta$. Inequality (2) specifies the lower bound $\left(\beta_{1}\right)$, and inequality (3) specifies the upper bound $\left(\beta_{2}\right)$ for $\beta$. The value of $\beta_{1}$ increases as the intensity ratio $\mathrm{Omax} / \mathrm{Omin}$ increases, and the value of $\beta_{2}$ decreases as the ratio Omax/Bmax decreases. Therefore, if $\beta_{1>}$ $\beta_{2}$ then perfect segmentation is not possible. Preprocessing the input image or enhancing the neuron model that effectively reduces $\beta_{1}$ and increases $\beta_{2}$ improves segmentation accuracy. Smoothing the image compresses the dynamic range of each region and also reduces the extent of intensity range overlap of regions. The net effect is a reduction in the value of $\beta_{1}$ and an increase in the value of $\beta_{2}$ as desired [3]. Kuntimad achieved improvement in segmentation accuracy by delaying the primary firing. This was accomplished by allowing the inactive neurons in the linking receptive field to send inhibitory linking inputs [4]. The approach is not biologically plausible, and also may not always improve segmentation. Note that delaying the primary firing to a value below Omax decreases values of both $\beta_{1}$ and $\beta_{2}$. This is beneficial only if the benefit of the reduction in the value of $\beta_{1}$ is relatively more than the harm caused by the reduction in the value of $\beta_{2}$.

Associated with the above approach, there are four major problems or issues which should be solved to further improve the segmentation accuracy of the PCNN approach.

1) The accuracy of the PCNN based algorithms is very sensitive to the values assigned to linking coefficient and linking neighbourhood radius. Almost always the linking radius is fixed at 1.5. Each neuron receives linking input from its 8neighbours. To the best of our knowledge the determination of the optimal value for the linking coefficient $\beta$ directly from the image is still an open problem that needs to be solved.

2) Previous experience with PCNN shows that a small change in the value of $\beta$ changes the segmentation result significantly. It is desirable to make the PCNN less sensitive to $\beta$.

3 ) It is very beneficial if one can delay primary firing to an appropriate intensity level between Omax and Bmax. This effectively reduces the lower bound for $\beta$ which is desirable. Also, prevents stray bright pixels from triggering primary firing.

4) When the PCNN is operated in the single-burst mode neurons corresponding to the background pixels are passive, and do not participate in the segmentation process. If object neurons attempt to capture a background neuron, background neurons do not oppose or resist capture. It is healthy if all neurons actively participate in the segmentation process. Therefore, there is a 
need to enhance the PCN model to include inhibitory linking input in a biologically plausible way.

\section{TWO-LAYER RECURRENT PCNN WITH EXCITATORY AND INHIBITORY LINKING}

This section presents a two-layer recurrent pulse coupled neural network with enhanced pulse coupled neuron model which has an additional linking receptive field called inhibitory receptive field. The new PCNN also operates in single-burst mode to segment tworegion images, and achieves higher image segmentation accuracy than the single-layer PCNN described in the previous section by resolving the four major problems identified above.

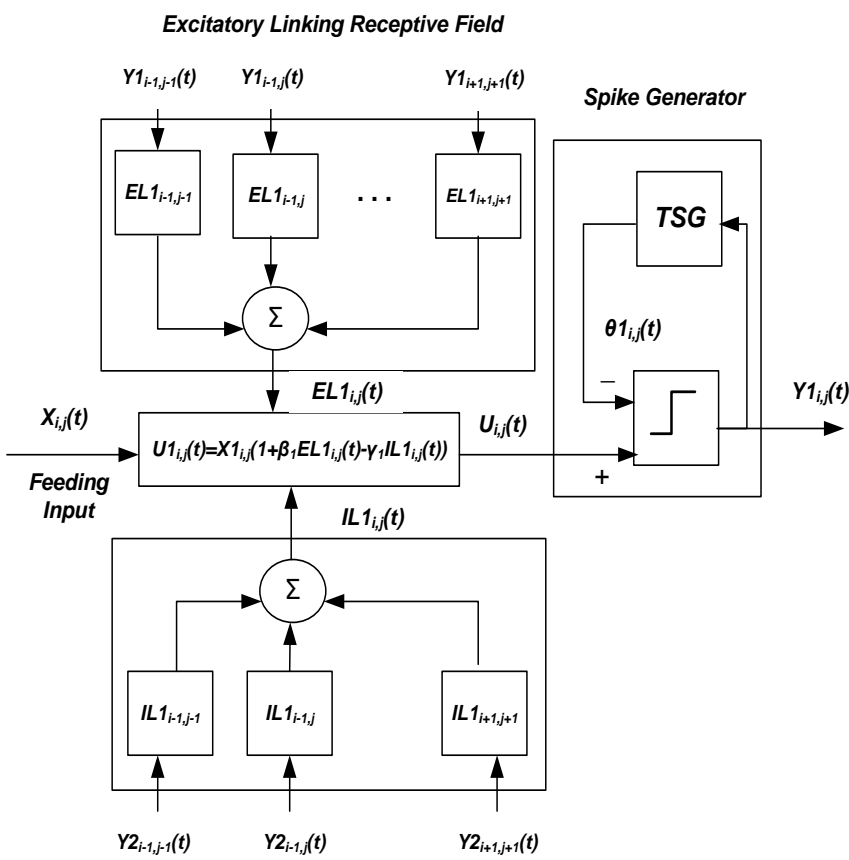

Inhibitory Linking Receptive Field

\section{Fig. 2 The enhanced PCN model}

The two-layer recurrent PCNN consists of two N X N laterally connected layers (Layer1 and Layer2) of pulse coupled neurons. The Layer1 neuron $N 1_{i, j}$ consists of a feeding input $X 1_{i, j}$ (intensity of image pixel $(i, j)$ ), an excitatory linking receptive field which gathers excitatory linking input $E L 1_{i, j}(t)$ from its 8-neighbors in Layer1, an inhibitory linking receptive field which gathers inhibitory linking input $I L 1_{i, j}(t)$ from the 8-neighbors of $N 2_{i, j}$ in Layer2, and a pulse generator as shown in Fig. 2. The internal activity of $N 1_{i, j}$ is computed by combining the feeding and linking inputs as

$$
U 1_{i, j}(t)=X 1_{i, j}\left(1+\beta_{1} E L 1_{i, j}(t)-\gamma_{1} I L 1_{i, j}(t)\right)
$$

where, $\beta_{1}>0$ and $\gamma_{1}>0$ are excitatory and inhibitory linking coefficients for Layer1. When $U 1_{i, j}(t)$ is greater than $\theta 1_{i, j}(t)$, the neuron $N 1_{i, j}$ fires $\left(Y 1_{i, j}(t)=1\right)$ and sends excitatory linking inputs to its 8-neighbors in Layer1, inhibitory linking inputs to its 8-neighbors in Layer2, and also charges the threshold signal generator in Layer1. Similarly, the internal activity of Layer2 neuron $N 2_{i, j}$ is computed as

$$
U 2_{i, j}(t)=X 2_{i, j}\left(1+\beta_{2} E L 2_{i, j}(t)-\gamma_{2} I L 2_{i, j}(t)\right)(5)
$$

where, $X 2_{i, j}$ is the feeding input, $E L 2_{i, j}(t)$ is the excitatory linking input from its 8-neighbors in Layer2, $I L 2_{i, j}(t)$ is the inhibitory linking inputs from the 8-neighbors of $N 1_{i, j}$ in Layer1, and $\beta_{2}>0$ and $\gamma_{2}>0$ are excitatory and inhibitory linking coefficients for Layer2.

The image segmentation approach using the two-layer PCNN is given in steps below:

1) For the image to be segmented, threshold $T$ which roughly segments the image into two regions (object and background) is determined. The threshold $T$ may be obtained using the basic iterative method, Otsu's method which maximizes inter-class variance or by locating the valley of the histogram if the histogram is bimodal. The intensity mean $O_{m}$ and standard deviation $O_{\sigma}$ of object pixels are approximated using image pixels with intensity greater than $T$. Similarly, the intensity mean $B_{m}$ and standard deviation $B_{\sigma}$ of background pixels are approximated using image pixels with intensity less than or equal to $T$.

2) The initial primary firing thresholds for Layer 1 neurons is taken as $T 1=\left(T+k O_{\sigma}\right)$. The linking coefficients $\beta_{1}$ and $\gamma_{1}$ should be computed such that the following inequality is satisfied.

Omin $\left(1+\beta_{1} \operatorname{EL1}(t)-\gamma_{1} I L 1(t)\right) \geq T 1$

Assume that $\beta_{1}=\gamma_{1}$, Omin is roughly equal to $\left(T-k O_{\sigma}\right)$, and linking is unity. Then the value of $(E L(t)-I L(t))$ may be taken as 2 assuming that the neuron corresponding to a boundary object pixel receives excitatory input from 5 neurons in Layer 1 and inhibitory linking input from 3 neurons in Layer2. Therefore,

$$
\beta_{1}=\gamma_{1}=\left(T 1 /\left(T-k O_{\sigma}\right)-1\right) / 2
$$

3) The inverted image is computed by subtracting the intensity of each pixel from 255 (highest possible intensity). The initial primary firing thresholds for Layer2 neurons which receive the inverted image as input is taken as $T 2=\left(255-T+k B_{\sigma}\right)$. The linking coefficients $\beta_{2}$ and $\gamma_{2}$ should be computed such that the following inequality is satisfied.

$(255-$ Bmax $)\left(1+\beta_{2} E L 2(t)-\gamma_{2} I L 2(t)\right) \geq T 2$

Assume that $\beta_{2}=\gamma_{2}$, Bmax is roughly equal to $\left(T+k B_{\sigma}\right)$, and linking is unity. Then the value of $(E L 2(t)-I L 2(t))$ may be taken as 2 assuming that the neuron corresponding to the boundary background pixel receives excitatory input from 5 neurons in Layer2 and inhibitory linking input from 3 neurons in Layer1. Therefore,

$$
\beta_{2}=\gamma_{2}=\left(T 2 /\left(255-T+k B_{\sigma}\right)-1\right) / 2
$$

4) The outputs of threshold signal generators in Layer1 and Layer2 are initialized to $T 1$ and $T 2$, respectively. The image to be segmented is applied as input to Layer1 and the inverted image is applied as input to Layer2, simultaneously.

5) Object neurons with feeding input greater than $T 1$ fire naturally in Layer1. Similarly, background pixels with feeding input greater than $T 2$ fire naturally in Layer2. The fired neurons initiate secondary firing to capture their neighbours in their own layer while attempt to prevent their neighbours from firing in the other Layer. If this recursive process is allowed to continue then the network will reach a stable state. The group of neurons firing together in Layer1 represent the object and other neurons represent the background. The group of neurons firing together in Layer2 represents the background and other neurons represent the object.

\section{SIMULATION RESULTS}

The segmentation performance of the two-layer PCNN is found to be significantly better than that of the optimal thresholding and single-layer PCNN, especially when region intensity ranges are wide 


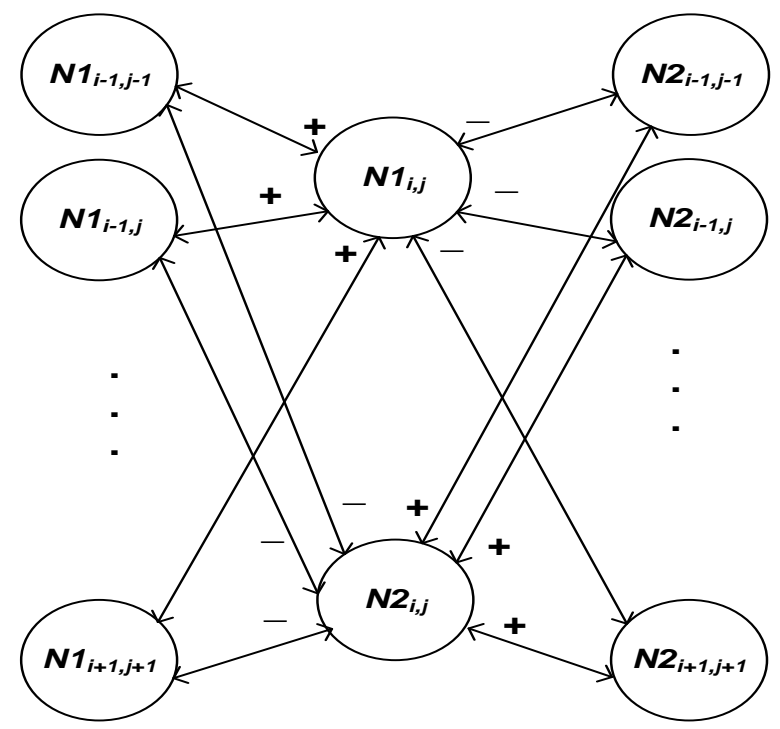

Fig. 3 Inter connection architecture for two-layer PCNN

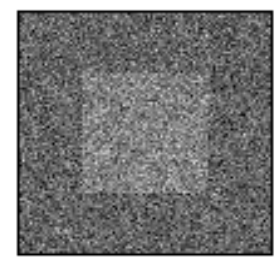

(a)

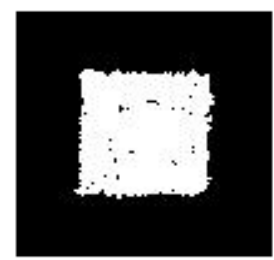

(c)

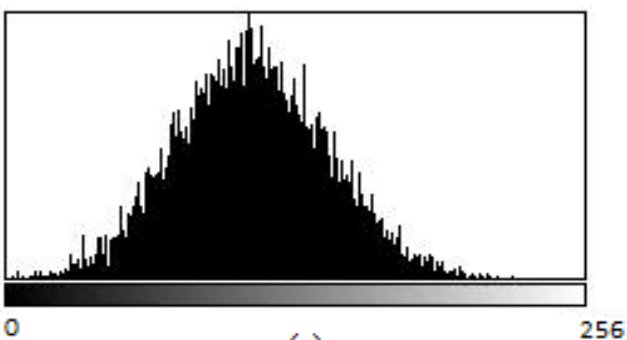

(e)

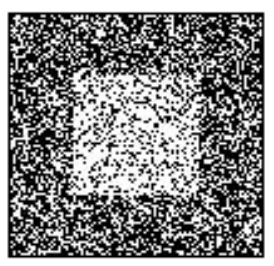

(b)

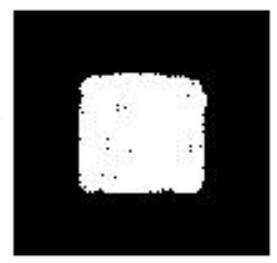

(d)
Fig. 4 Example 1. (a) The input image. (b) Segmentation result from Otsu's optimal thresholding method. (c) Segmentation result from single-layer PCNN. (d) Segmentation result from two-layer PCNN.

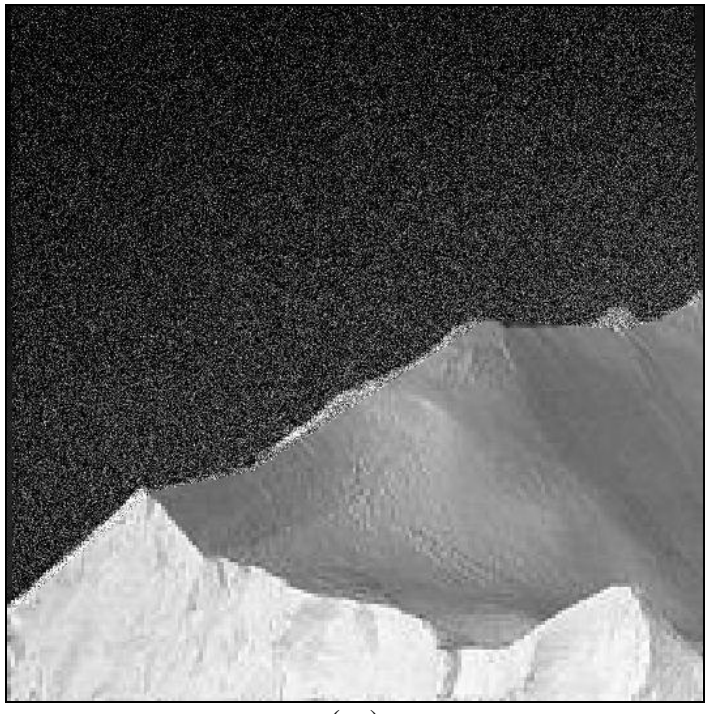

( a )

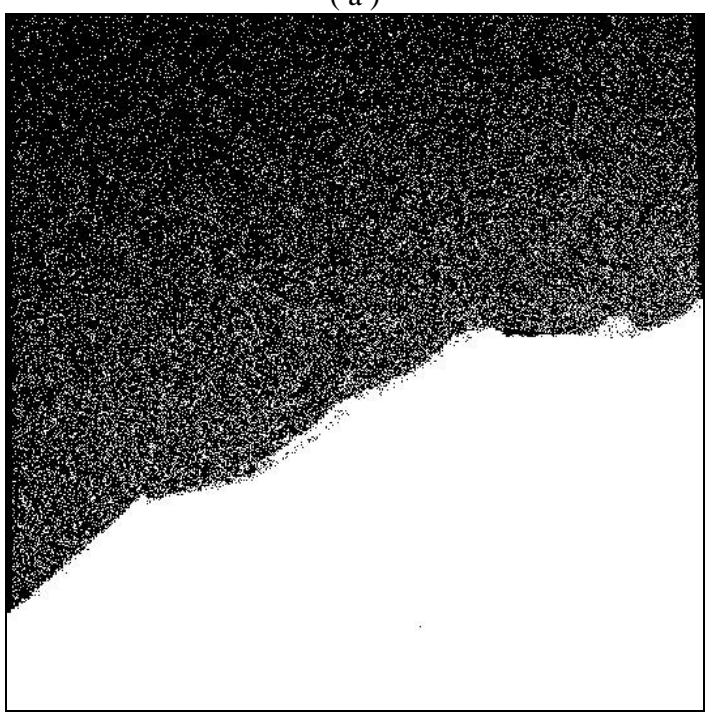

( b )

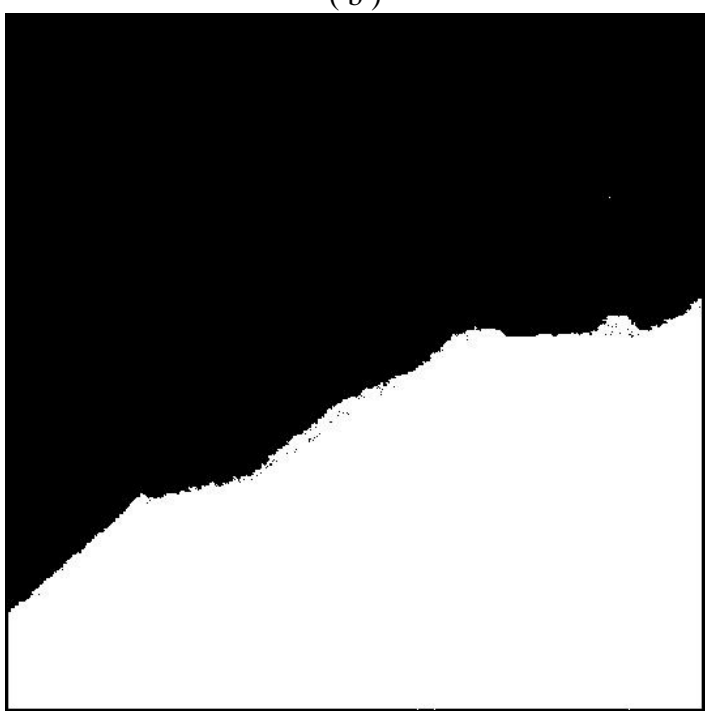

(c) 


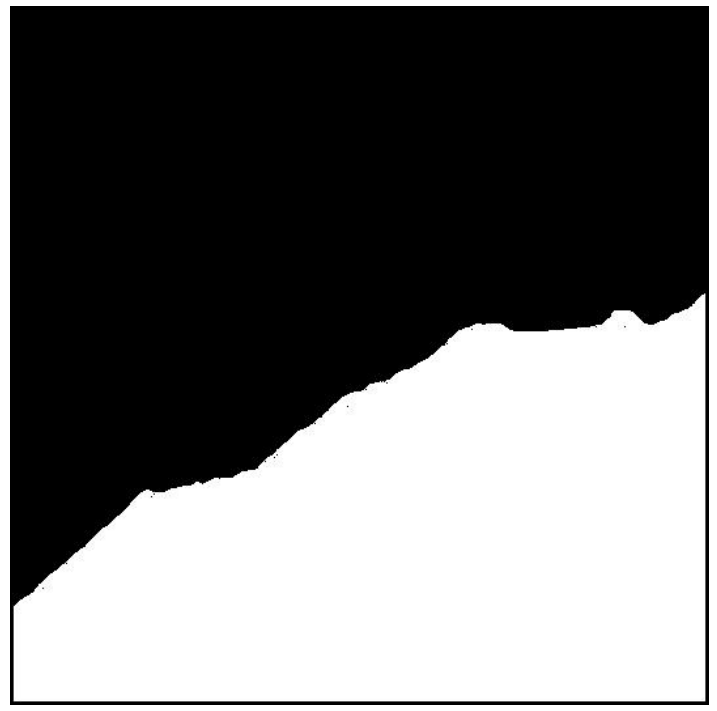

( d )

Fig. 5 Example 2. (a) A noisy image of an iceberg. (b) Segmentation result from Otsu's optimal thresholding method. (c) Segmentation result from single-layer PCNN. (d) Segmentation result from twolayer PCNN.

and the extent of their overlap is large. The image in Fig. 4 consists of two regions. The intensity range, mean, and variance of object pixels are [28, 227], 130, and 31, respectively. The intensity range, mean, and variance of background pixels are [11, 200], 100, and 31, respectively. From the image histogram in Fig. 4(b), it is obvious that this is not an easy image to segment. As expected, Otsu's optimal thresholding method which determines threshold by maximizing inter-class variance failed miserably as shown in Fig. 4 (c). A similar result was obtained when Bayes optimal threshold was used. Kuntimad's single layer PCNN performed remarkably well as can be seen from the segmented image given in Fig. 4(d). However, it is important to note that the value of the linking coefficient $\beta(0.45)$ was determined by trial and error to achieve the best performance. When $\beta$ was set to 0.4 , more object pixels were classified as background pixels, and when $\beta$ was set to 0.5 , more background pixels were captured as object pixels during secondary firing. The two-layer recurrent PCNN was able to segment the image much better than the single-layer PCNN. The values of all parameters ( $T 1$, $T 2, \beta_{1}, \beta_{2}$,) were directly determined from the image histogram as described in Section IV. The value of $k$ was kept constant at 1 . Note that the object edges are sharp and practically there is no bleeding of the object into the background area. When the value of $\mathrm{k}$ was varied from 0.5 to 1.5 , there was almost no change in the performance of the network. As a second example, the iceberg image in Fig. 5(a) was segmented using Otsu's method, Kuntimad's single-layer PCNN and the two-layer PCNN. As expected, the two-layer PCNN gave almost perfect result. The segmented images for the three methods are given in Fig. 5(b), Fig. 5(c) and Fig. 5(d), respectively.

\section{COMPARISON OF SINGLE-LAYER PCNN WITH TWO- LAYER PCNN}

In this section, single-layer PCNN and two-layer PCNN are compared based on segmentation accuracy and the sensitivity to network parameters (primary firing threshold and linking coefficient). The image in Fig. 4(a) is used in all experiments. The image is segmented using single-layer PCNN by varying the linking coefficient from 0.16 to 0.48 in steps of 0.02 while the primary firing threshold is held constant at 190. Table I shows the number of object and background pixels incorrectly classified, and the overall percent error. Note that the minimum error of $2.3 \%$ is achieved when $\beta$ is 0.34 . A $50 \%$ deviation in the value of $\beta$ from its optimal value results in an order of magnitude increase in the number of pixels misclassified illustrating that the segmentation accuracy is highly sensitive to $\beta$.

TABLE I

EFFECT OF LINKING COEFFICIENT ON SEGMENTATION ACCURACY

\begin{tabular}{|c|c|c|c|}
\hline \multirow[t]{2}{*}{$\beta$} & \multicolumn{2}{|c|}{ Number of misclassified pixels } & \multirow[t]{2}{*}{ Percent error } \\
\hline & Object & Background & \\
\hline 0.16 & 2207 & 21 & 22.28 \\
\hline 0.18 & 2117 & 24 & 21.41 \\
\hline 0.2 & 1877 & 27 & 19.04 \\
\hline 0.21 & 1348 & 30 & 13.78 \\
\hline 0.23 & 750 & 41 & 7.91 \\
\hline 0.25 & 461 & 66 & 5.27 \\
\hline 0.27 & 273 & 87 & 3.6 \\
\hline 0.3 & 107 & 127 & 2.34 \\
\hline 0.32 & 73 & 158 & 2.31 \\
\hline 0.34 & 54 & 176 & 2.3 \\
\hline 0.36 & 40 & 270 & 3.1 \\
\hline 0.38 & 28 & 359 & 3.87 \\
\hline 0.4 & 25 & 558 & 5.83 \\
\hline 0.42 & 18 & 897 & 9.15 \\
\hline 0.44 & 14 & 1242 & 12.56 \\
\hline 0.46 & 12 & 2267 & 22.79 \\
\hline 0.48 & 9 & 3623 & 36.32 \\
\hline
\end{tabular}

The simulation results in Table II show that setting the primary firing threshold $T$ to an appropriate value below Omax usually improves the segmentation accuracy. When the primary firing threshold is set to 220, best result is obtained. In other words, using Omax as the primary firing threshold as suggested by Kuntimad may not yield good segmentation, if the object includes a few bright stray pixels.

In the two-layer PCNN, the primary firing thresholds and the linking coefficients are functions of parameter $k$. Therefore, effectively the number of parameters is reduced to one. Also, the segmentation accuracy is found to be less sensitive to the variation of $k$ from its optimal value as shown in Table III. A 50\% change in the value of $k$ from its optimal value of 0.4 increases the error from $1.2 \%$ to $1.63 \%$ or $2.35 \%$, depending on the direction of change.

In two-layer PCNN, the primary firing threshold is set to a relatively low value as compared to the value used in the single-layer PCNN. As a result, many background neurons fire during primary firing along with object neurons. However, due to inhibitory input from the Layer2, most of the background neurons get turned off at a later time during the recurrent operation. From Table IV, it is clear 
that 167 object neurons did not fire and 90 background neurons fired during primary firing. After 8 iterations network reached a stable state. In the stable state, overall percent error was 1.18 (97 object and 21 background pixels were misclassified).

TABLE II

EFFECT OF PRIMARY FIRING THRESHOLD ON SEGMENTATION ACCURACY

\begin{tabular}{|l|l|l|l|l|}
\hline \multirow{2}{*}{\begin{tabular}{l}
$\boldsymbol{T}$ \\
\multirow{2}{*}{$\boldsymbol{\beta}$}
\end{tabular}} & \multicolumn{2}{|l|}{$\begin{array}{l}\text { Number of pixels } \\
\text { Misclassified }\end{array}$} & \multirow{2}{\text{Percent}}{ error } \\
\cline { 3 - 4 } & & Object & Background & \\
\hline 225 & 0.58 & 19 & 558 & 5.77 \\
\hline 220 & 0.42 & 79 & 80 & 1.59 \\
\hline 215 & 0.42 & 50 & 115 & 1.65 \\
\hline 210 & 0.4 & 50 & 115 & 1.65 \\
\hline 205 & 0.36 & 86 & 87 & 1.73 \\
\hline 200 & 0.34 & 82 & 105 & 1.87 \\
\hline 195 & 0.34 & 70 & 128 & 1.98 \\
\hline 190 & 0.34 & 54 & 176 & 2.3 \\
\hline
\end{tabular}

TABLE III

MISCLASSIFIED PIXELS FOR VARYING VALUES OF $k$

\begin{tabular}{|l|l|l|l|l|l|l|}
\hline $\mathbf{k}$ & T1 & T2 & $\boldsymbol{\beta}_{\boldsymbol{1}}$ & $\boldsymbol{\beta}_{\boldsymbol{2}}$ & \multicolumn{2}{|l|}{ Misclassified Pixels } \\
\cline { 6 - 7 } & & & & & Object & Background \\
\hline 0.2 & 113 & 150 & 0.02 & 0.014 & 167 & 68 \\
\hline 0.3 & 115 & 152 & 0.03 & 0.022 & 115 & 41 \\
\hline 0.4 & 117 & 154 & 0.04 & 0.029 & 97 & 21 \\
\hline 0.5 & 119 & 156 & 0.053 & 0.037 & 126 & 15 \\
\hline 0.6 & 121 & 158 & 0.064 & 0.045 & 158 & 5 \\
\hline 0.7 & 124 & 160 & 0.077 & 0.054 & 177 & 0 \\
\hline 0.8 & 126 & 162 & 0.09 & 0.062 & 196 & 0 \\
\hline
\end{tabular}

TABLE IV

RECURRENT OPERATION OF THE TWO-LAYER PCNN

\begin{tabular}{|l|l|l|}
\hline \multirow{2}{*}{ Iteration } & Misclassified Pixels \\
\cline { 2 - 3 } & Object & Background \\
\hline 0 & 167 & 90 \\
\hline 1 & 102 & 52 \\
\hline 2 & 95 & 37 \\
\hline 3 & 95 & 32 \\
\hline 4 & 97 & 28 \\
\hline 5 & 97 & 24 \\
\hline 6 & 97 & 23 \\
\hline 7 & 97 & 21 \\
\hline 8 & 97 & 21 \\
\hline
\end{tabular}

\section{CONCLUSION}

This paper has presented a two-layer recurrent Pulse Coupled Neural Network which used a modified Pulse Coupled Neuron with an inhibitory linking receptive field. The new PCNN has adequately addressed and resolved the four major issues associated with the single-layer PCNN described in Section II. The network parameters are directly computed from the image histogram eliminating the need for the trial-and-error method used by the single-layer PCNN. The use of two layers, inhibitory receptive fields, and the recurrent operation has reduced the sensitivity to network parameters. It is important to note that all neurons participate in the segmentation process as desired. In summary, the segmentation accuracy of the two-layer recurrent PCNN is significantly better than the segmentation accuracy of the single-layer PCNN operating in single-burst mode.

\section{REFERENCES}

[1] R. Eckhorn, H.J. Reitboeck, M. Arndt, and P.W. Dicke, "Feature linking vis synchronization among distributed assemblies: Simulation results from cat visual cortex and from simulations," in Neural comput., Vol 2, pp.293-307, 1990.

[2] H. S. Ranganath, G. Kuntimad, and J. L. Johnson, "pulse coupled neural networks for image processing," IEEE Southeastcon, Raleigh, NC, Mar. 1995.

[3] G. Kuntimad, "Pulse coupled neural networks for image processing," Ph.D. dissertation, Department of Computer Science, The University of Alabama in Huntsville, 1995.

[4] G. Kuntimad and H.S. Ranganath, "Perfect segmentation using pulse coupled neural networks," IEEE Transactions on Neural networks, Vol. 10, No. 3, pp. 591-598, 1999.

[5] J. A. Karvonen, "Baltic sea ice SAR segmentation and classification using modified pulse coupled neural networks," IEEE Transactions on Geoscience and Remote Sensing, Vol 42, No. 7, pp. 1566-1574, 2004.

[6] R. D. StewartI. Fermin, and M. Opper, "Region growing with pulse coupled neural networks: an alternative to seeded region growing. IEEE Transactions on Neural Networks, Vol. 13, No. 6, pp.1557-1562, 2002.

[7] Y. Ma, R. Dai, and L. Li, "Image segmentation of embryonic plant cell using pulse coupled neural networks," Chinease Science Bulletin, Vol. 47, No. 2, pp. 167-172, 2002.

[8] Y. Ma, Q. Liu, and Z. Quian, "Automated image segmentation using improved PCN model based on cross-entropy," Journal of Image and Graphics, Vol. 10, pp. 579-584, 2005.

[9] Xiao, Shi, and Chang, "Automatic image segmentation based on PCNN and fuzzy mutual information," IEEE Ninth International Conference on Computer and Information Technology, pp. 241-245, 2009.

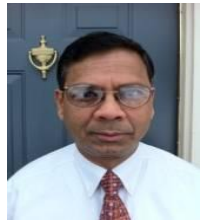

Heggere Ranganath received his Ph.D. degree in Electrical Engineering from Auburn University in 1980. Since 2002 he is serving as the chair of the Computer Science Department at the University of Alabama in Huntsville, Huntsville, Alabama. During his 30-year career as a professor, Dr. Ranganath has served as a technical advisor to many private technology companies and Government agencies, received over $\$ 4,000,000$ in research funding, and published over 100 technical papers. His areas of research include Image Processing, Pattern Recognition, and Artificial Neural Networks.

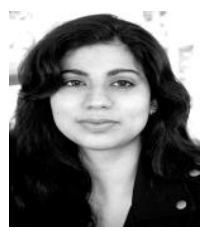

Ayesha Bhatnagar received her Bachelor of Engineering degree from Mumbai University, Mumbai, India. After earning her Master of Science degree in Computer Science, she is currently pursuing her Ph.D. degree in Computer Science at the University of Alabama in Huntsville, Huntsville, Alabama. Image Processing and Artificial Neural Networks are her areas of research interest 